\title{
Neonatal Nutritional Deprivation or Enhancement: The Cardiac-Sympathetic Axis and its Role in Cardiac Growth and Stress Responses
}

\author{
JOANNE M. BELL, WILLIAM L. WHITMORE, AND THEODORE A. SLOTKIN \\ Department of Pharmacology, Duke University Medical Center, Durham, North Carolina 27710
}

\begin{abstract}
To determine the mechanisms by which neuronal input influences cardiac growth during altered neonatal nutritional status, rats were reared in small, standard, or large litter sizes and the adrenergically mediated stimulation of cardiac ornithine decarboxylase was determined; ornithine decarboxylase provides a mechanistic link connecting adrenergic input to cardiac growth. Nutritionally deprived pups showed impaired development of sympathetic reflex stimulation as shown by the attenuation of the cardiac ornithine decarboxylase response to hydralazineinduced hypotension throughout the preweanling period. The subnormal reactivity to hydralazine reflected a defect in neurotransmission, as a full response was obtained with $\operatorname{direct} \beta$-receptor stimulation (isoproterenol). Nevertheless, cardiac hypertrophy in response to repeated isoproterenol administration was markedly suppressed in nutritionally deprived animals, suggesting that the $\beta$-receptor/ornithine decarboxylase pathway had become uncoupled from growth. Because maturation of neural connections to peripheral tissues causes a loss of hypoxia tolerance, nutritional status also influenced the ability of neonatal rats to survive hypoxia. These data indicate that cardiac growth suppression or enhancement caused by nutritional manipulations may be mediated, in part, through alterations in the development of neuronal input to the tissue, and that similar factors influence survival during hypoxic stress. (Pediatr Res 23: 423-427, 1988)
\end{abstract}

\section{Abbreviations}

ODC, ornithine decarboxylase ANOVA, analysis of variance sc, subcutaneous

Neonatal food restriction results in a constellation of pathophysiological changes in which the central nervous system is spared most of the insult but peripheral tissues are not (1-3). Recent data in the rat suggest that the vulnerability of specific tissues to nutritional insult is coordinated through early biochemical signals involving the ODC polyamine pathway. ODC initiates the synthesis of putrescine, spermidine, and spermine, which are now recognized as major controllers of macromolecule synthesis during periods of cell replication and rapid growth (4-6).

In the heart, a tissue whose growth rate is directly dependent on nutritional status, ODC activity is influenced by sympathetic neuronal input acting via $\beta$-adrenergic receptors $(7-10)$. The

Received September 14, 1987; accepted December 15, 1987.

Correspondence Dr. Joanne M. Bell, Box 3813, Duke University Medical Center Durham, NC 27710.

Supported by USPHS Grants HD-09713 and NS-06233. cardiac-sympathetic axis develops in three stages (11). At birth, receptors are present but sympathetic input is low or absent; by the end of the first postnatal week, sympathetic efferent structures develop, permitting transmission of signals which are dependent on metabolic status (including blood sugar levels); during the second and third postnatal weeks, afferent sensory information from the baroreceptors begins to regulate sympathetic tone, with a resultant increase in impulse flow. The different stages play a significant role in cardiac cell replication and differentiation, as the early period of low tonic sympathetic activity has a promotional effect on cell acquisition and growth (10), whereas the later surge in tone terminates replication and initiates growth by cell enlargement $(7,9)$.

Accordingly, our study was designed to test the hypothesis that the control of neonatal cardiac growth in response to nutritional alterations is exerted, in part, through effects on development of sympathetic innervation and/or its coupling to growth. We have examined the development of the $\beta$-receptor-mediated ODC response to direct (isoproterenol) or central reflex (hydralazineinduced hypotension) stimulation, along with the ability of $\beta$ receptor stimulation to cause cardiac hypertrophy. Finally, as the development of innervation plays a role in developmental shifts in the ability to maintain cardiac function during hypoxia, we have determined whether nutritional status affects hypoxic survival time in the presence and absence of neural input.

\section{METHODS}

Primiparous Sprague-Dawley rats (Zivic-Miller Laboratories, Allison Park, PA) were shipped by climate-controlled truck 2 wk before parturition and were placed on Purina Rat Chow (RalstonPurina Co., St. Louis, MO) ad libitum. Immediately after birth, pups from all litters were randomized and redistributed into three categories: five to six pups/litter (small litter), 10-11 pups/ litter (standard litter), or 16-17 pups/litter (large litter). As pups were chosen for each experiment, randomization within each category was carried out again in order to maintain the respective litter sizes. Animals of both sexes were randomly selected from different cages on each experimental day and groups were always sex-matched. Pups were weighed, killed by decapitation, and tissues removed and weighed before biochemical analyses.

Cardiac biochemical response to sympathetic stimulation. Development of baroreceptor-mediated sympathetic stimulation of the myocardium was assessed by measuring the ability of hydralazine, a vasodilator, to elicit an increase in heart ODC activity (12). Animals were given $3.3 \mathrm{mg} / \mathrm{kg}$ of hydralazine sc every 20 min for a total of six injections; controls received saline. Animals were killed $4.5 \mathrm{~h}$ after the first injection and hearts removed for assay of ODC.

In another series of experiments, animals were given a direct $\beta$-adrenergic receptor agonist, $l$-isoproterenol $\mathrm{HCl}(0.5 \mathrm{mg} / \mathrm{kg} \mathrm{sc})$ daily beginning at 15 days of postnatal age and continuing 
through 20 days postnatally (13). Four $\mathrm{h}$ after the first injection and $4 \mathrm{~h}$ after the last injection, animals were killed, hearts weighed to assess the degree of isoproterenol-induced cardiac hypertrophy, and the tissues were then analyzed for ODC activity.

For the determination of ODC activity, hearts were homogenized in 19-39 volumes of $10 \mathrm{mM}$ Tris (pH 7.2) and the samples were centrifuged at $26,000 \times g$ for $15 \mathrm{~min}$. Enzyme activity was then determined in aliquots of the supernatant as described previously (14), using a final concentration of $5 \mu \mathrm{M}\left[{ }^{14} \mathrm{C}\right]$ ornithine.

Survival during hypoxia. To determine survival during hypoxic conditions, animals were placed in a covered 22-liter clear plastic pan which was maintained at $37^{\circ} \mathrm{C}$ and ventilated at a rate of $15 \mathrm{liter} / \mathrm{min}$ with a warmed, humidified mixture of $5 \% \mathrm{O}_{2}+$ $95 \% \mathrm{~N}_{2}$. Survivors were counted every $15 \mathrm{~min}$ for up to 150 min. In one experiment, 4-day-old animals were pretreated with chlorisondamine chloride $(5 \mathrm{mg} / \mathrm{kg}$ sc given $20 \mathrm{~min}$ before commencing hypoxia), a nicotinic receptor antagonist; this dose of chlorisondamine has been shown previously to be completely effective in developing rats (15).

Statistics. Parametric data are presented as means \pm SE, with significance evaluated by ANOVA with factors of litter size and age. Nonparametric variables were compared using Fisher's exact test. Significance was evaluated with criteria of $p<0.05$ and $p<$ 0.01 .

Materials. Chlorisondamine chloride and hydralazine were obtained from Ciba Pharmaceuticals (Summit, NJ) and $l$-isoproterenol $\mathrm{HCl}$ from Sigma Chemical Corp. (St. Louis, MO). L-[1${ }^{14} \mathrm{C}$ ]ornithine (specific activity $52 \mathrm{mCi} / \mathrm{mmol}$ ) was purchased from New England Nuclear Corp., Boston, MA.

\section{RESULTS}

Body and heart growth (Fig. 1). Nutritional deprivation or enhancement produced by alterations in litter size had a profound effect on growth of neonatal rats. Both the nutritionally enhanced and nutritionally deprived groups diverged from the standard litter group beginning at $1 \mathrm{wk}$ of age and the differences intensified thereafter. Heart weight was affected similarly. In both cases, differences in growth remained present through the immediate postweaning period.

Responses to sympathetic stimulation (Fig. 2). Stimulation of heart ODC activity by hydralazine reached a peak during the second postnatal week in pups reared in standard litters, corresponding to the approximate age at which baroreceptor reflexes become established and are initially hyperresponsive $(16,17)$. Although overnutrition had no net facilitatory action on either the magnitude or age course of development of the response, undernutrition severely attenuated the effect on ODC at all ages and blunted the peak of responsiveness.

In contrast to the findings with hydralazine, acute administration of isoproterenol at 15 days of age successfully evoked stimulation of ODC activity in the undernourished group and the effect was indistinguishable from either the overnourished or standard animals. After daily administration of isoproterenol, stimulation by the drug in all three nutritional groups remained of the same magnitude on the last day of treatment (day 20). Cardiac hypertrophy caused by the repeated injections of isoproterenol was assessed on day 20 and showed equivalent degrees of hypertrophy in the overnourished and normally nourished group, but severely restricted hypertrophy in the malnourished animals.

Survival during hypoxia (Fig. 3). In general, survival during severe hypoxia showed the expected loss of tolerance with age: nearly all animals survived for more than $100 \mathrm{~min}$ at $5 \% \mathrm{O}_{2}$ at 4 days of age regardless of litter size, but by 30 days of age, all animals died within $20 \mathrm{~min}$. However, between these extremes there were substantial differences in hypoxic survival capabilities caused by nutritional alterations. Even by 4 days of age, before significant changes in body growth characteristics have become apparent, the overnourished animals displayed greater mortality during hypoxia than did the standard or undernourished pups; there was, however, no advantage to undernourishment at this time, because these animals reared in large litters died at the same rate as (or even slightly faster than) the standard group. By 6 days of age, differences appeared among all three groups, intensifying with the development of weight differences, so that by 15 days of age all the overnourished animals died within 45 min, only half the animals died in the standard litter group and none of the animals died in the malnourished cohort. All differences in sensitivity disappeared by 30 days of age, despite the maintenance of body weight disparities.

The role of neurogenic input in surviving hypoxia was evaluated at 4 days of age, at which time the transition to mature efferent sympathetic function is underway $(11,15)$. Pretreatment with chlorisondamine to block neural input changed the hypoxic survival characteristics substantially from the pattern seen without the drug. Animals in all three groups died more quickly during hypoxia after drug treatment and the mortality curves for the overnourished and standard pups became indistinguishable from each other. In contrast, the undernourished animals pretreated with chlorisondamine died at a slower rate than did those
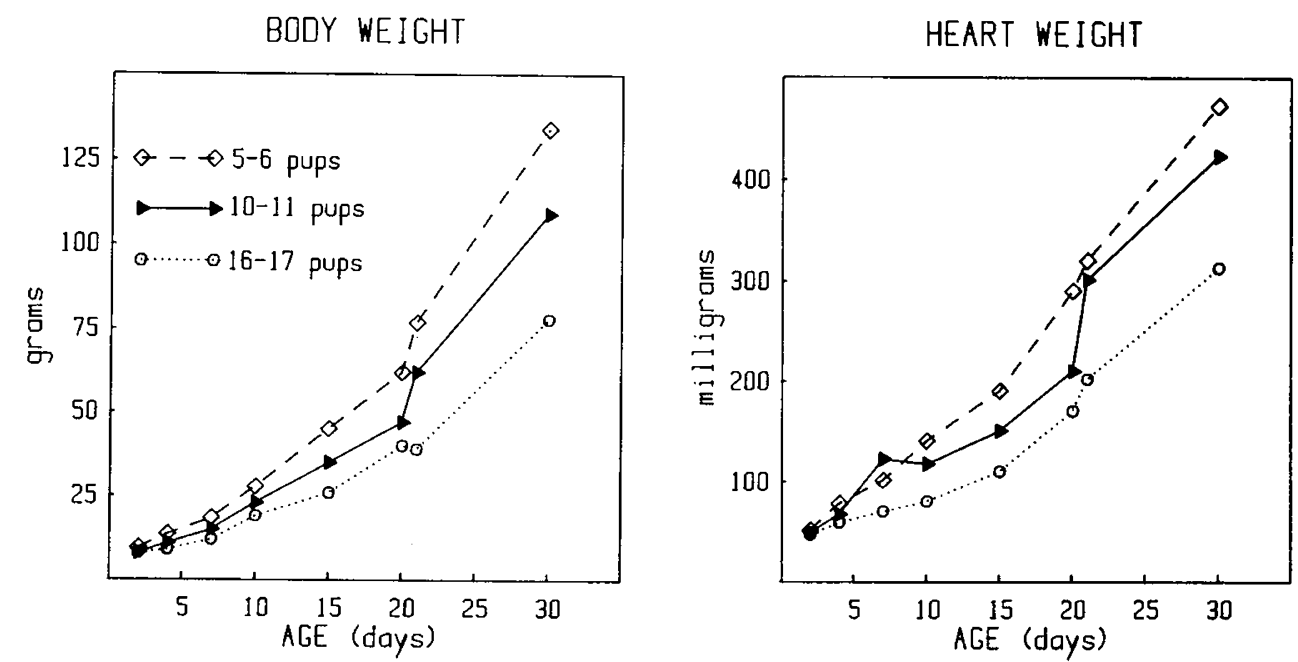

Fig. 1. Effects of litter size manipulations on development of body and heart weight. Data represent values from at least eight animals selected from different litters in each group at each age. SE $<3 \%$. Two-way ANOVA indicates a significant main effect of litter size $(p<0.01)$ as well as a litter size $\times$ age interaction $(p<0.01)$ for both variables. 


\section{ACUTE HEART DOC STIMULATION \\ BY HYDRALAZINE}

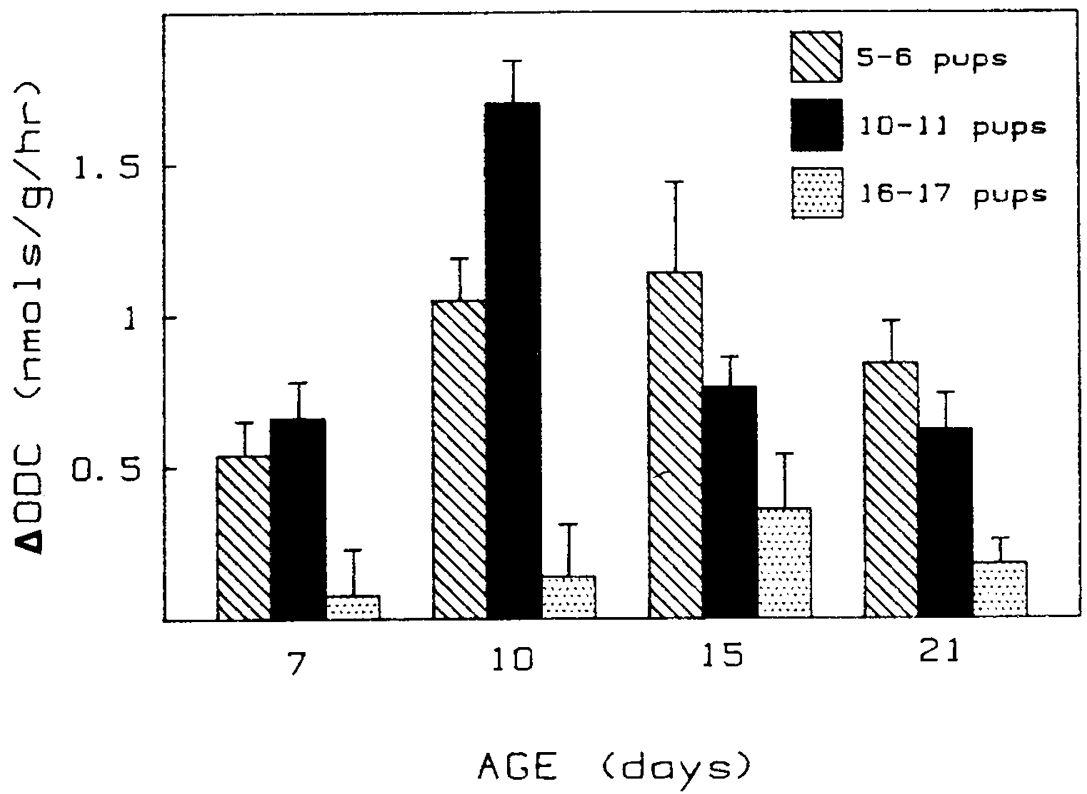

\section{ACUTE HEART ODC STIMULATION BY JSOPROTERENOL}
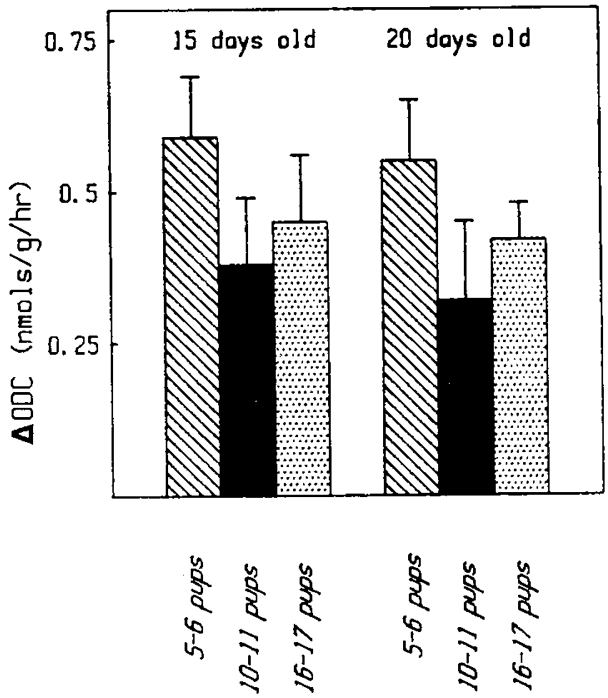

ISOPROTERENOL-INDUCED CARDIAC HYPERTROPHY

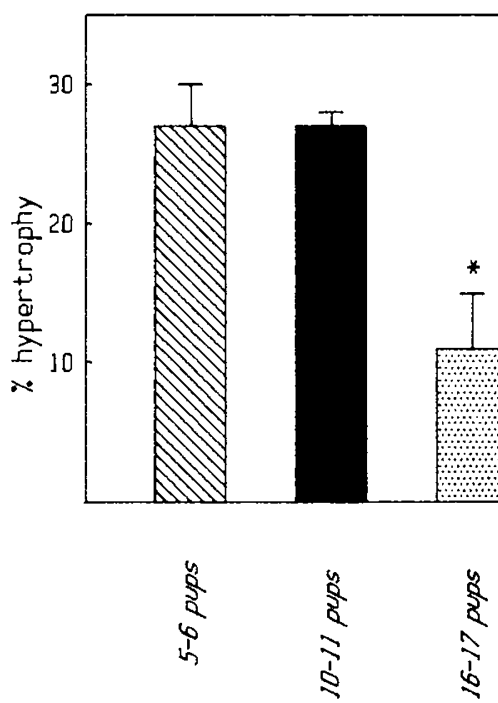

Fig. 2. Stimulation of cardiac ODC activity after hydralazine (top) or isoproterenol (bottom left), and cardiac hypertrophy caused by 5 days of daily treatment with isoproterenol (bottom right). For ODC stimulation, animals were given the drug treatments as described in "Methods," and killed 4 to $4.5 \mathrm{~h}$ later. Data represent means and SE of values obtained from five to eight animals in each treatment group. $\triangle$ ODC activity was calculated from the difference between values in stimulated animals versus basal activities, which were: for five to six pups/litter, $0.78 \pm 0.10 \mathrm{nmol} /$ $\mathrm{g} / \mathrm{h}$ at 7 days, $0.75 \pm 0.04$ at 10 days, $0.76 \pm 0.04$ at 15 days, and $0.62 \pm 0.04$ at $20-21$ days; for $10-11$ pups/litter, $0.63 \pm 0.06,0.81 \pm 0.01,0.81$ $\pm 0.01,0.56 \pm 0.04$; for $16-17$ pups/litter, $1.05 \pm 0.15,1.22 \pm 0.11,0.73 \pm 0.09,0.60 \pm 0.09$. Percent hypertrophy was calculated from the difference in heart/body weight ratio between animals given chronic isoproterenol versus chronic saline; basal values were: $0.0046 \pm 0.0001$ for five to six pups/litter, $0.0047 \pm 0.0006$ for 10-11 pups/litter, and $0.0043 \pm 0.0003$ for 16-17 pups/litter. For hydralazine stimulation of ODC, ANOVA indicates a significant overall reduction of effect in the 16-17 pups/litter group $(p<0.01)$ and a significant litter size $\times$ age interaction $(p<0.05)$. Stimulation of ODC by isoproterenol was unaffected by age or litter size. Cardiac hypertrophy caused by isoproterenol was significantly attenuated in the $16-17 \mathrm{pups} /$ litter category $(p<0.01)$.

in the other two groups and were now clearly distinguishable from the standard size animals.

\section{DISCUSSION}

The current results support the view that effects on peripheral sympathetic function contribute to the biochemical events ac- companying cardiac growth restriction during nutritional deprivation. Earlier studies have shown that sympathetic input, acting through the ODC/polyamine system, regulates the level of cardiac DNA, RNA, and protein synthesis during neonatal development and during subsequent hypertrophy $(3,9,10,14)$. It is particularly important, then, that neuronal input to the heart is 
4 DAYS OLD

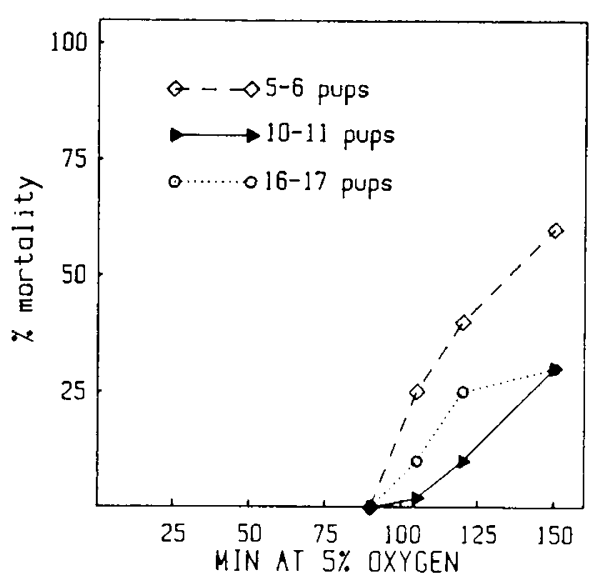

6 DAYS OLD

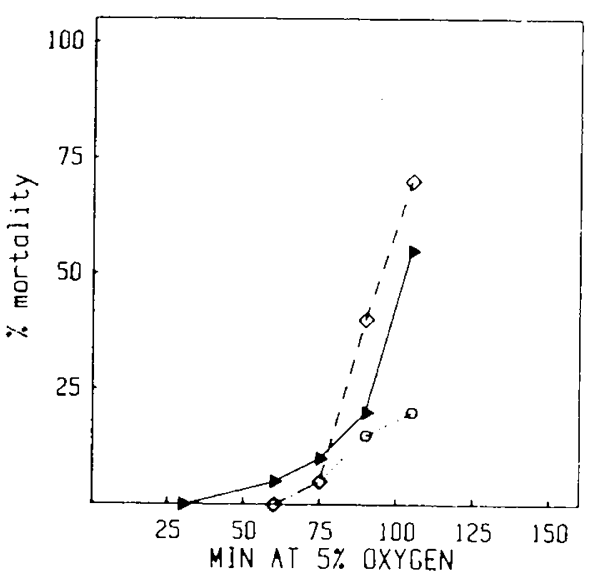

15 DAYS OLD

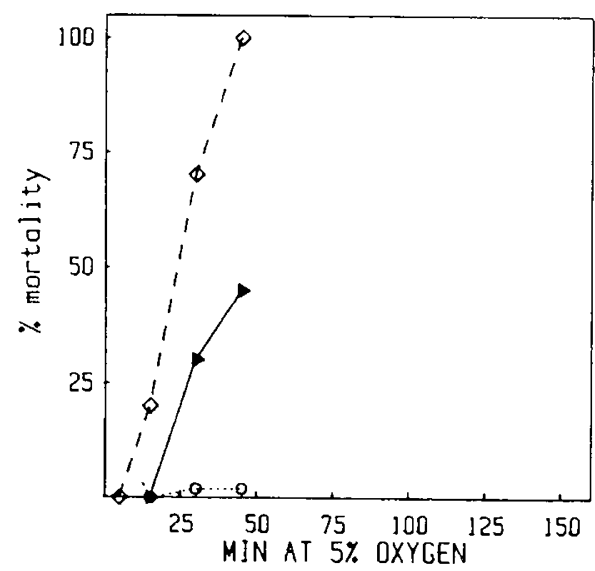

4 DAYS OLD

CHLORISONDAMINE PRETREATED

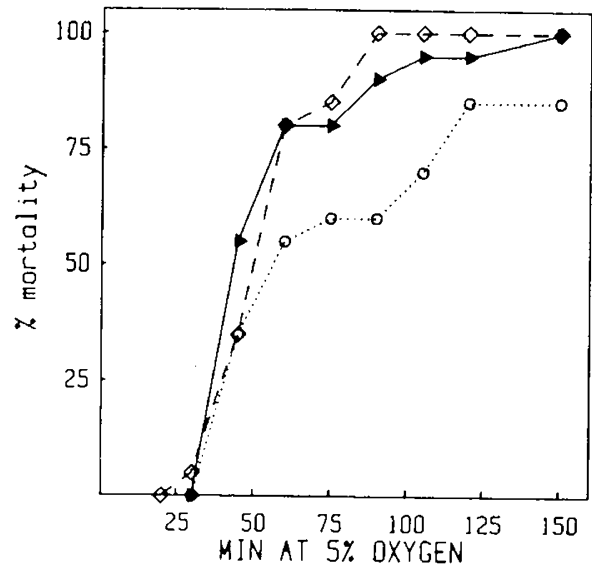

9 DAYS OLO

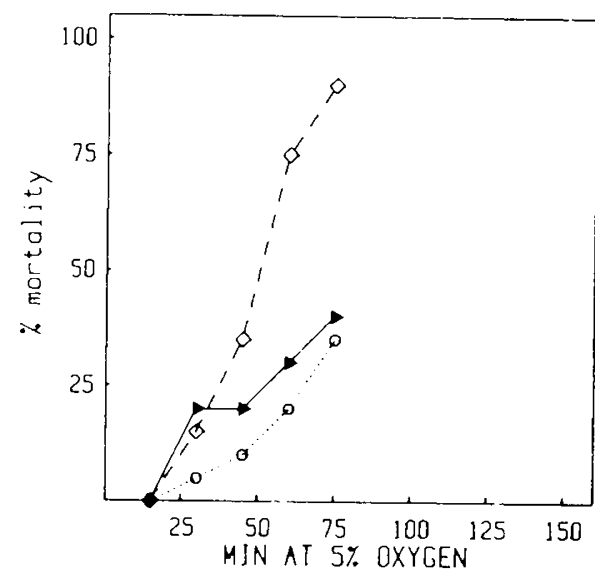

30 DAYS OLD

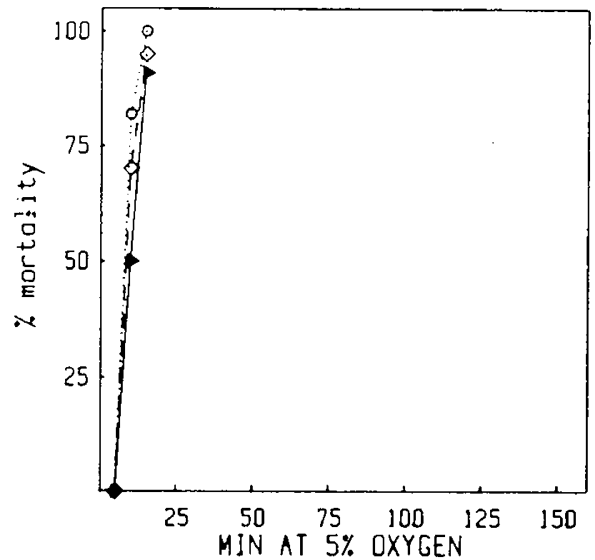

Fig. 3. Mortality data during exposure to $5 \% \mathrm{O}_{2}$ at various ages. Values were obtained from at least 40 pups in each litter size group at each age. For animals receiving no drug pretreatment, Fisher's exact test indicates significantly overall higher mortality rates in the overnourished group $(p<$ $0.01)$. Undernourished animals survived significantly better overall than those reared in standard size litters $(p<0.01)$. With chlorisondamine pretreatment, 4-day-old animals in all groups died significantly faster $(p<0.01)$ than did their respective untreated cohorts; in addition, undernourished animals pretreated with chlorisondamine died more slowly than did treated animals in the other two groups $(p<0.01)$. 
suppressed in the malnourished state, as evidenced by the lowered reactivity of ODC to baroreceptor-mediated cardiac sympathetic stimulation (hydralazine). Because the ODC response to isoproterenol was unaffected, the reduced efficacy of hydralazine most likely represents an effect on neural maturation or activity. Second, the fact that isoproterenol-induced cardiac hypertrophy was suppressed in malnourished rats suggests that the $\beta$-receptorinduced stimulation of ODC becomes uncoupled from cardiac growth, with or without concurrent changes in the number of $\beta$ receptors. Thus, both neuronal and postreceptor loci are involved in the adjustment of the cardiac-sympathetic axis to conditions of impaired nutrition.

These factors play a less important role in the case of overnutrition. The ODC response to reflex (hydralazine) or direct (isoproterenol) $\beta$-receptor stimulation was nearly equivalent in overnourished animals and in animals reared in standard litters. Similarly, the growth response to chronic sympathetic stimulation (isoproterenol-induced hypertrophy) was not enhanced by overfeeding. Nevertheless, basal ODC activity and polyamine levels (without acute stimulation) have been shown to be elevated in nutritionally enhanced animals (3), indicating that in this circumstance a major contribution to growth is provided by nonneuronal signals. One likely possibility is insulin, which also influences cardiac ODC and which would certainly be elevated in animals experiencing overfeeding (18). Because overfeeding enhances sympathetic neuronal catecholamine levels (19), these findings raise the possibility that sympathetic input may be permissive to development, and that once a minimum neuronal signal is obtained, other factors predominate in further growth.

The onset of sympathetic innervation also plays a role in functional responses of the cardiovascular system. Survival during neonatal hypoxia, for example, requires release of adrenal catecholamines which occurs even in the absence of neural signals, and the nonneurogenic response disappears with the onset of synaptic activity $(15,20)$. Neonatal cardiac conduction can be maintained for prolonged periods during hypoxia, but only if the adrenal catecholamines can stimulate cardiac $\alpha$ adrenergic receptors; if the receptors are blocked, death ensues from cardiac arrest $(17,21)$. The fetal and neonatal heart is unusually enriched in $\alpha$-receptors, a condition that also disappears with the onset of innervation $(22,23)$. We, therefore, examined whether nutritional status influenced survival during hypoxia. Indeed, malnourished rats survived better than did the normal group or the overnourished animals. It is unlikely that this difference is purely metabolic or size related: differences were detectable at 4 days of age, before any major growth enhancement or restriction had developed, and disappeared at 30 days when the animals were all eating ad libitum but still had different weights. Studies conducted at one age point suggest that the differences may reflect, at least during the $1 \mathrm{st} \mathrm{wk}$, the slowing of development of neural input to the adrenal and/or heart. At 4 days of age, a point at which neural function is becoming established in normal rats (11), survival was virtually indistinguishable between the malnourished group and those reared in normal-size litters. However, when animals were pretreated with chlorisondamine to block neural input, the growth-restricted animals survived hypoxia much better than did those experiencing normal nutrition, indicating that the normal group had become more dependent on neurally mediated responses.

In conclusion, these results show that the restriction of tissue growth during neonatal malnutrition is not a passive event but rather reflects a careful coordination of trophic signals that regulate organ development. In the case of the heart, neural stimulation and postsynaptic transduction of receptor activation provide at least two sites at which this control is expressed. Similar factors during under- or overnutrition may operate to influence physiological responses that determine survival during severe stresses such as hypoxia.

\section{REFERENCES}

1. Dodge PR, Prensky AL, Feigin RD 1975 Nutrition and the Developing Nervous System. CV Mosby Company, St. Louis, MO

2. Parvez S, Ishmahan G, Parvez H 1980 Role of nutritional factors in the development of catecholamine synthesis and metabolism. In: Parvez S, Parvez H (eds) Biogenic Amines in Development. Elsevier, Amsterdam, pp 441-492

3. Bell JM, Whitmore WL, Queen KL, Orband-Miller L, Slotkin TA 1987 Biochemical determinants of growth sparing during neonatal nutritional deprivation or enhancement: ornithine decarboxylase, polyamines, and macromolecules in brain regions and heart. Pediatr Res 22:599-604

4. Heby O 1981 Role of polyamines in the control of cell proliferation and differentiation. Differentiation 19:1-20

5. Russell DH 1985 Ornithine decarboxylase: a key regulatory enzyme in normal and neoplastic growth. Drug Metab Rev 16:1-88

6. Slotkin TA, Bartolome J 1986 Role of ornithine decarboxylase and the polyamines in nervous system development: a review. Brain Res Bull 17:307320

7. Claycomb WC 1976 Biochemical aspects of cardiac muscle differentiation: Possible control of deoxyribonucleic acid synthesis and cell differentiation by adrenergic innervation and cyclic adenosine $3^{\prime}: 5^{\prime}$-monophosphate. $\mathrm{J}$ Biol Chem 251:6082-6089

8. Deskin R, Mills E, Whitmore WL, Seidler FJ, Slotkin TA 1980 Maturation of sympathetic neurotransmission in the rat heart. VI. The effect of neonatal central catecholaminergic lesions. J Pharmacol Exp Ther 215:342-347

9. Slotkin TA, Whitmore WL, Orband-Miller L, Queen KL, Haim K $1987 \beta$ Adrenergic control of macromolecule synthesis in neonatal rat heart, kidney and lung: relationship to sympathetic neuronal development. J Pharmacol Exp Ther 243:101-109

10. Slotkin TA, Levant B, Orband-Miller L, Queen KL, Stasheff S 1988 Do sympathetic neurons coordinate cellular development in the heart and kidney? Effects of neonatal central and peripheral catecholaminergic lesions on cardiac and renal nucleic acids and proteins. J Pharmacol Exp Ther (in press)

11. Slotkin TA 1986 Endocrine control of synaptic development in the sympathetic nervous system. The cardiac-sympathetic axis. In: Gootman PM (ed) Developmental Neurobiology of the Autonomic Nervous System. Humana Press, Clifton NJ, pp 97-133

12. Bartolome J, Mills E, Lau C, Slotkin TA 1980 Maturation of sympathetic neurotransmission in the rat heart. V. Development of baroreceptor control of sympathetic tone. J Pharmacol Exp Ther 215:596-600

13. Bartolome JV, Trepanier PA, Chait EA, Slotkin TA 1982 Role of polyamines in isoproterenol-induced cardiac hypertrophy: effects of $\alpha$-difluoromethylornithine, an irreversible inhibitor of ornithine decarboxylase. $\mathrm{J}$ Mol Cell Cardiol 14:461-466

14. Bartolome J, Huguenard J, Slotkin TA 1980 Role of ornithine decarboxylase in cardiac growth and hypertrophy. Science 210:793-794

15. Seidler FJ, Slotkin TA 1985 Adrenomedullary function in the neonatal rat: responses to acute hypoxia. J Physiol 358:1-16

16. Seidler FJ, Slotkin TA 1981 Development of central control of norepinephrine turnover and release in the rat heart: responses to tyramine, 2-deoxyglucose and hydralazine. Neuroscience 6:2081-2086

17. Smith PG, Slotkin TA, Mills E 1982 Development of sympathetic ganglionic neurotransmission in the neonatal rat. Pre- and postganglionic nerve response to asphyxia and 2-deoxyglucose. Neuroscience 7:501-507

18. Aubert R, Suquet J-P, Lemonnier D 1979 Long-term morphological and metabolic effects of early under- and over-nutrition in mice. J Nutr 110:649661

19. Heggeness FW, Diliberto J, DeStefano V 1970 Effect of growth velocity on cardiac norepinephrine content in infant rats. Proc Soc Exp Biol Med 133:1413-1416

20. Seidler FJ, Slotkin TA 1986 Ontogeny of adrenomedullary responses to hypoxia and hypoglycemia: Role of splanchnic innervation. Brain Res Bull $16: 11-14$

21. Seidler FJ, Brown KK, Smith PG, Slotkin, TA 1987 Toxic effects of hypoxia on neonatal cardiac function in the rat: $\alpha$-Adrenergic mechanisms. Toxicol Lett 37:79-84

22. Noguchi A, Whitsett JA, Dickman $\mathbf{L} 1981$ Ontogeny of myocardial $\alpha_{1}$ receptor in the rat. Dev Pharmacol Ther 3:179-188

23. Slotkin TA, Kavlock RJ, Cowdery T, Orband L, Bartolome M, Whitmore WL, Bartolome J 1986 Effects of neonatal methylmercury exposure on adrenergic receptor binding sites in peripheral tissues of the developing rat. Toxicology $41: 95-106$ 